\title{
Effects of Lignin-Derived Polycarboxylic Acids on the Properties of Waterborne Polyurethane Elastomers
}

\author{
Ruiqi Wang $\mathbb{D}^{1}{ }^{1}$ Bing Zhou, ${ }^{1}$ Yanchao Zhu $\mathbb{D}^{1,2}$ and Zichen Wang ${ }^{1}$ \\ ${ }^{1}$ College of Chemistry, Jilin University, Qianjin Street 2699, Changchun 130012, China \\ ${ }^{2}$ Institute for Frontier Materials, Deakin University, Geelong, VIC 3216, Australia \\ Correspondence should be addressed to Yanchao Zhu; yanchao_zhu@jlu.edu.cn
}

Received 11 May 2018; Revised 31 July 2018; Accepted 26 August 2018; Published 4 October 2018

Academic Editor: Cheng Zhang

Copyright (c) 2018 Ruiqi Wang et al. This is an open access article distributed under the Creative Commons Attribution License, which permits unrestricted use, distribution, and reproduction in any medium, provided the original work is properly cited.

\begin{abstract}
Lignin was modified by oxidation to prepare lignin-derived polycarboxylic acids (LPCAs), which can be introduced into waterborne polyurethane (WPU) elastomers through the interactions with the soft segments of WPU. The changes of water resistance, thermal and tensile properties of WPU elastomers were observed. After LPCAs were added, the water solubility of WPU elastomers was increased. Besides, the weight loss peak of the soft segments of WPU moved to the lower temperature, indicating that the decomposition of soft segments became easier and the interspace of the soft segments regions was enlarged by LPCAs. Comparing with the blank WPU elastomer, when the LPCA loading was $2.5 \mathrm{wt} \%$ (based on PPG-1000), the tensile stress of the LPCA-WPU materials at $100 \%$ and $300 \%$ strain increased $135.4 \%$ and $90.5 \%$ separately, and the modulus of elasticity in tension increased $383.6 \%$. Therefore, LPCAs could serve as reinforcing fillers in WPU elastomers, which contributes to develop more functional and eco-friendly WPU materials. Moreover, the usage of lignin is broadened.
\end{abstract}

\section{Introduction}

Polyurethane is a widely used polymeric material for its performance can be adjusted in a large range by applying different polyols, isocyanates, catalysts, and auxiliary compounds [1]. In our everyday life, polyurethane is used in various fields like adhesives, coatings, elastomers, and foams [2]. Among them, waterborne polyurethane (WPU) is more popular because it is eco-friendly [3]. To improve the properties of WPU, various functional materials, like cellulose $[4,5]$, xanthan [6], graphene and its oxide $[7,8]$, nanofibers $[9,10]$, and nanoparticles $[11,12]$, have been introduced into WPU to form composites. Besides, the introducing method of functional materials also affects the properties of WPU. In general, there are three methods to introduce: at the stage of chain-extending, in the midst of emulsification, and at the post of emulsification [13]. Different introducing methods can bring about different modification effects as well.

Lignin, an important bioresource, is the most abundant aromatic polymer in nature [14]. Through different isolation processes, the original lignin was converted to other types like kraft lignin, lignosulfonate, organosolv lignin, and steam explosion lignin [15]. With several functional groups such as carboxyl, methoxyl, and hydroxyl [16], lignin can be chemically modified by different methods like alkylation, hydroxymethylation, Mannich reaction, oxidation, and phenolation $[17,18]$. Therefore, lignin can be used to prepare polyurethane composites, phenol-formaldehyde resins, epoxide resins, biomedical materials, etc. $[19,20]$. As a modification method of lignin, oxidation is also a promising way to obtain valuable products [15]. Many reagents, like nitrobenzene, hydrogen peroxide, metal oxides, and laccase, have been used to oxidize lignin and the corresponding applications have been achieved $[20,21]$. For example, kraft lignin could be oxidized by hydrogen peroxide and the oxidization products were employed as a dispersant for kaolin suspensions [22]. Utilizing oxidative products of lignin contributes to exploit the bioresource lignin and to develop more high value applications $[23,24]$.

In this work, we took advantage of lignin-derived polycarboxylic acids (LPCAs) to modify the properties of WPU 
elastomers. $\mathrm{NaOCl}$ solution, an oxidative reagent which can be operated under mild conditions, was chosen to perform oxidative modification on steam explosion lignin to prepare LPCAs. LPCAs were introduced into WPU in the midst of emulsification. We discussed the modification mechanism of the LPCA-WPU elastomers and observed the changes of the water resistance, thermal and mechanical properties of LPCA-WPU elastomers.

\section{Materials and Methods}

2.1. Materials. Steam explosion lignin was donated by Jilin KAIYU Biomass Development \& Utilization Co. Ltd. Polypropylene glycol-1000 (PPG-1000) was obtained from Jiangsu Haian Petrochemical Works. Tolylene-2,4diisocyanate (TDI) was supplied by Tianjin Fuchen Chemical Reagents Factory. 2,2-Dimethylolpropionic acid (DMPA) was provided by Chengdu Gracia Chemical Technology Co. Ltd. All the other reagents used in this study were purchased in China and were of analytical grade. The concentration of $\mathrm{NaOCl}$ solution was calibrated according to the China National Standard GB 19106-2003.

2.2. Preparation of LPCAs. $5.00 \mathrm{~g}$ steam explosion lignin and $150 \mathrm{~mL}$ distilled water were placed into a $500 \mathrm{~mL}$ three-neck round-bottom flask. The mixture in the flask was heated to $30^{\circ} \mathrm{C}$ in a water bath and was stirred mechanically. Then, $\mathrm{NaOCl}$ solution was added at a ratio $[n(\mathrm{NaOCl}) / m($ lignin $)]$ of $12 \mathrm{mmolg}^{-1}$ in $10 \mathrm{~min}$. The total time of stirring was $30 \mathrm{~min}$. Afterwards, $6 \mathrm{~mol} \mathrm{~L}^{-1} \mathrm{HCl}$ was added to achieve a $\mathrm{pH}$ of 2 (using $\mathrm{pH}$ test strips with a range of 1 to 14 ). The precipitate was centrifuged, washed once with distilled water, dried at $80^{\circ} \mathrm{C}$ for $24 \mathrm{~h}$, and grounded to obtain LPCAs. To compare the effect of oxidative modification, some amount of acid-precipitated lignin was prepared from steam explosion lignin.

2.3. Synthesis of LPCA-WPU Elastomers. The typical process of the synthesis of LPCA-WPU elastomers was as follows. $0.10 \mathrm{~g}$ LPCAs and $10 \mathrm{~mL}$ distilled water were added into a $100 \mathrm{~mL}$ beaker, and then $2 \mathrm{~mol} \mathrm{~L}^{-1} \mathrm{NaOH}$ solution was added to make LPCAs just dissolved. The solution of LPCAs was diluted to $75 \mathrm{~mL}$ with distilled water to be ready for the synthesis of LPCA-WPU elastomers.

$10.00 \mathrm{~g}$ PPG-1000 was added into a $500 \mathrm{~mL}$ four-neck round-bottom flask and nitrogen gas was purged continuously. Under mechanical stirring, $4.36 \mathrm{~g}$ TDI was added into the flask at room temperature. After that, the temperature of the water bath was raised to $70^{\circ} \mathrm{C}$ and the mixture was stirred for $2 \mathrm{~h}$. Next, the temperature of the water bath was reduced to $60^{\circ} \mathrm{C}$. $0.94 \mathrm{~g}$ DMPA, $50 \mathrm{~mL}$ acetone, and 2 drops of dibutyltin dilaurate were added successively. The reaction was carried out for $2 \mathrm{~h}$. During the reaction, acetone would be added additionally to lower the viscosity of the mixture. When the reaction finished, the temperature of the water bath was reduced to $40^{\circ} \mathrm{C}$ and $0.70 \mathrm{~g}$ triethylamine was fed into the flask. The mixture was stirred for $10 \mathrm{~min}$. And then, the solution of LPCAs was added and the stirring was sped up properly and kept for $1 \mathrm{~h}$. Later, the water emulsion
TABLE 1: The titration analysis and GPC characterization results of acid-precipitated lignin and LPCAs.

\begin{tabular}{lcc}
\hline & Acid-precipitated lignin & LPCAs \\
\hline$w(\mathrm{COOH})$ & $9.02 \%$ & $11.10 \%$ \\
$M_{\mathrm{n}}$ & $2.91 \times 10^{4}$ & $1.23 \times 10^{4}$ \\
$M_{\mathrm{w}}$ & $3.48 \times 10^{4}$ & $1.25 \times 10^{4}$ \\
\hline
\end{tabular}

was poured into a mold and dried at $60^{\circ} \mathrm{C}$ for $48 \mathrm{~h}$ to get the elastomer. Then the elastomer was put into a sample bag and was kept at room temperature for 1 week to be ready for characterization.

The LPCA loading in LPCA-WPU elastomers is expressed as the weight ratio of LPCAs vs PPG-1000, i.e., $m$ (LPCAs)/ $m(\mathrm{PPG}-1000) \times 100 \%$, and its value was set as $0 \mathrm{wt} \%, 1 \mathrm{wt} \%$, $1.5 \mathrm{wt} \%, 2.5 \mathrm{wt} \%, 5 \mathrm{wt} \%$, and $7.5 \mathrm{wt} \%$, respectively.

2.4. Characterization of LPCAs. The content of carboxyl was determined by titration analysis [25]. $200 \mathrm{mg}$ LPCAs and $20 \mathrm{~mL} 0.4 \mathrm{~mol} \mathrm{~L}^{-1}$ calcium acetate solution were mixed in a $100 \mathrm{~mL}$ round-bottom flask. The mixture was heated at $85^{\circ} \mathrm{C}$ for $30 \mathrm{~min}$. Then, it was cooled to room temperature and filtrated. The filtrate was transferred into a $50 \mathrm{~mL}$ volumetric flask and was diluted by distilled water to reach the graduation. $20 \mathrm{~mL}$ distilled water and $10 \mathrm{~mL}$ solution in the volumetric flask were added into a $250 \mathrm{~mL}$ conical flask. $0.05 \mathrm{~mol} \mathrm{~L}^{-1} \mathrm{NaOH}$ solution was used in titration with phenolphthalein as the indicator. Blank test was performed meanwhile, in which the sample was not used. The content of carboxyl was calculated according to (1):

$$
w(\mathrm{COOH})=\frac{\left(V_{1}-V_{0}\right) \times c \times 5 \times 45.018}{m} \times 100 \%,
$$

where $V_{1}$ and $V_{0}$ were the volumes of $\mathrm{NaOH}$ solution that the sample and the blank test consumed separately; $c$ was the concentration of the $\mathrm{NaOH}$ solution; 5 was the conversion factor, i.e., $50 \mathrm{~mL} / 10 \mathrm{~mL} ; 45.018$ was the molar weight of carboxyl; $m$ was the weight of the sample in the roundbottom flask.

The molecular weight of lignin was measured by gel permeation chromatography (GPC) using Agilent PL-GPC 220. The temperature of the column was $70^{\circ} \mathrm{C}$. The type of the column was PLgel.

2.5. Characterization of LPCA-WPU Elastomers. The water resistance test was carried out in room temperature. A sample with an area of approximately $1 \mathrm{~cm}^{2}$ was cut from each elastomer. Later, the samples were placed into distilled water for $12 \mathrm{~h}$. Afterwards, the samples were taken out and were wiped up by filter paper, and then the final weight of the samples were measured. The percentage of the weight change was figured out according to (2):

$$
W=\frac{m_{2}-m_{1}}{m_{1}} \times 100 \%,
$$

where $m_{1}$ was the initial weight of the sample and $m_{2}$ was the final weight of the sample. 


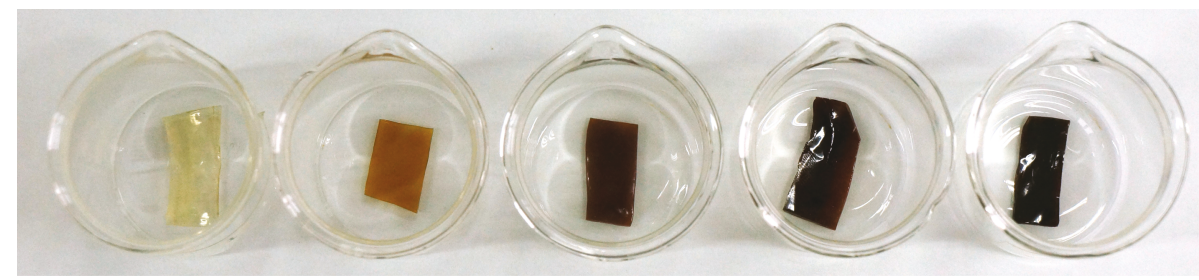

(a)

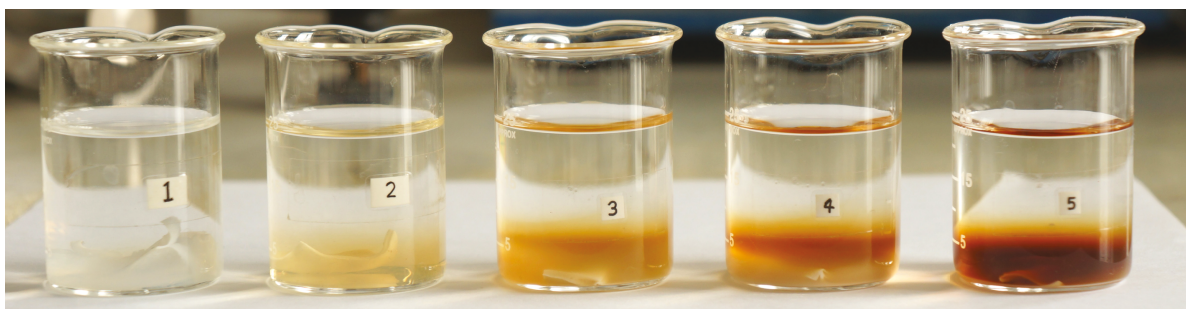

(b)

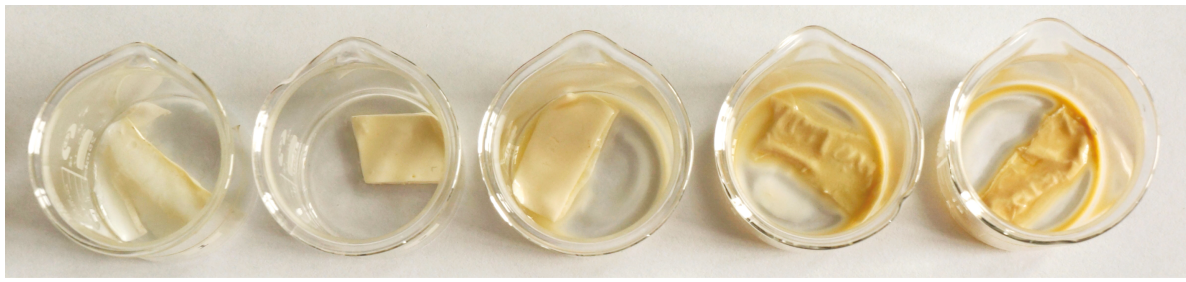

(c)

Figure 1: The photos of LPCA-WPU elastomers immersed in water after $12 \mathrm{~h}$.

The thermogravimetric analysis was performed by a thermal analyzer (NETZSCH STA 449C) under a nitrogen atmosphere from 40 to $800^{\circ} \mathrm{C}$ at a heating rate of $10^{\circ} \mathrm{C} \mathrm{min}{ }^{-1}$.

The tensile performance was characterized by a microcomputer-controlled electronic universal testing machine (Jinan Liangong Testing Technology Co. Ltd. CMT-20) with a tensile rate of $100 \mathrm{~mm} \mathrm{~min}^{-1}$ according to the China National Standard GB/T 1040-2006. The temperature of the room for the tensile test was kept as $23^{\circ} \mathrm{C}$ and the relative humidity was kept as $50 \%$ according to the China National Standard GB/T 2918-1998. The elastomers were cut into strips with dumbbell-like shapes, of which the width of the central part was $4 \mathrm{~mm}$. Both ends of the strips were clamped tightly in the tensile test to just let the central part of them stretched. For each elastomer, at least 5 strips were used to calculate the average value and the standard deviation.

\section{Results and Discussion}

3.1. Characterization Results of Lignin. The content of carboxyl, number-average molecular weight $\left(M_{\mathrm{n}}\right)$, and weightaverage molecular weight $\left(M_{\mathrm{w}}\right)$ of acid-precipitated lignin and LPCAs are shown in Table 1.

After lignin was oxidized by $\mathrm{NaOCl}$ solution, the content of carboxyl increased and the molecular weight decreased, indicating that LPCAs were prepared successfully.

3.2. Water Resistance of LPCA-WPU Elastomers. Figure 1 shows the result of water resistance test of LPCA-WPU
TABLE 2: The percentage of the weight change of LPCA-WPU elastomers with different loadings of LPCAs.

\begin{tabular}{lc}
\hline LPCA loading & $W$ \\
\hline $0 \mathrm{wt} \%$ & $167.8 \%$ \\
$1.0 \mathrm{wt} \%$ & $102.2 \%$ \\
$2.5 \mathrm{wt} \%$ & $53.9 \%$ \\
$5.0 \mathrm{wt} \%$ & $-22.9 \%$ \\
$7.5 \mathrm{wt} \%$ & $-42.3 \%$ \\
\hline
\end{tabular}

elastomers and Table 2 shows the percentage of the weight change of the elastomers. In Figure 1, from the left to the right, the LPCA loading of the elastomers is $0 \mathrm{wt} \%, 1.0 \mathrm{wt} \%$, $2.5 \mathrm{wt} \%, 5.0 \mathrm{wt} \%$, and $7.5 \mathrm{wt} \%$ separately. Figure $1(\mathrm{a})$ is the photo of the elastomers before distilled water was added. After the elastomers were immersed in distilled water for $12 \mathrm{~h}$, the result is shown in Figure 1(b). Then, the liquid in each beaker was removed and the remnant is shown in Figure 1(c). As LPCAs were dissolved in $\mathrm{NaOH}$ solution prior to being added to WPU, the more LPCAs were added, the more $\mathrm{NaOH}$ would be needed. When LPCA-WPU elastomers were immersed in water, the elastomers would absorb water and the weight of them would increase. As the time of immersing went on, $\mathrm{NaOH}$ was dissolved in water and started to dissolve LPCAs again. As a result, the weight of the elastomers would decrease. With the increase of LPCA loading, the total amount of LPCAs and $\mathrm{NaOH}$ increased while the percentage of the weight change of LPCA-WPU 


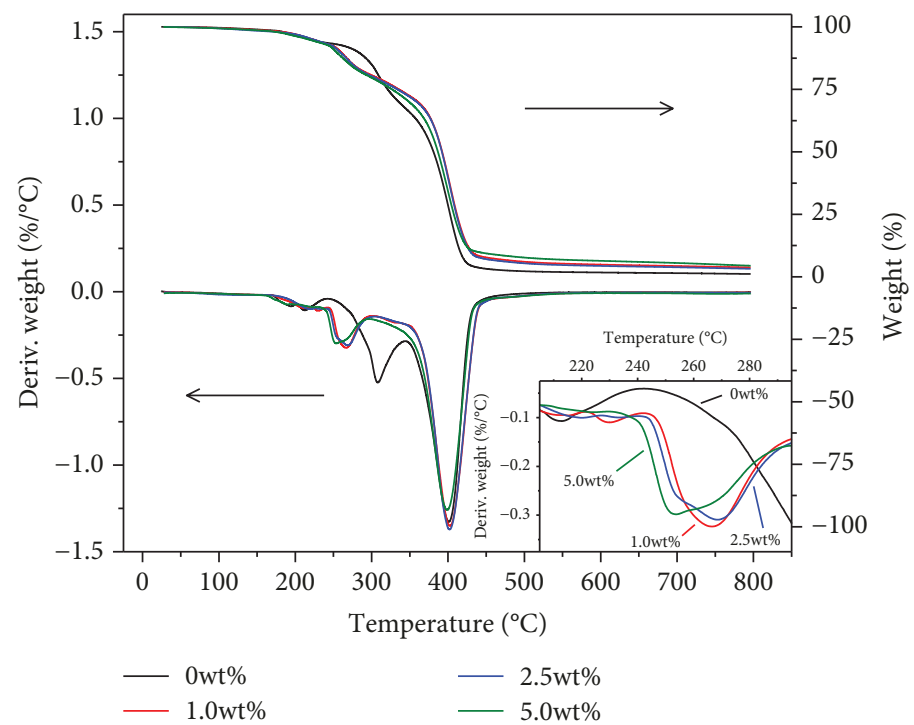

FIGURE 2: TG and DTG curves of LPCA-WPU elastomers with different loadings of LPCAs.

TABLE 3: Weight percentage of the residue $\left(w_{\mathrm{r}}\right)$, temperature of the weight loss peak of the soft segments $\left(t_{\mathrm{SS}}\right)$ and the hard segments $\left(t_{\mathrm{HS}}\right)$ of LPCA-WPU elastomers with different loadings of LPCAs.

\begin{tabular}{lccc}
\hline LPCA loading & $w_{\mathrm{r}}$ & $t_{\mathrm{SS}}\left({ }^{\circ} \mathrm{C}\right)$ & $t_{\mathrm{HS}}\left({ }^{\circ} \mathrm{C}\right)$ \\
\hline $0 \mathrm{wt} \%$ & $1.132 \%$ & 308.06 & 401.14 \\
$1.0 \mathrm{wt} \%$ & $3.782 \%$ & 266.41 & 402.54 \\
$2.5 \mathrm{wt} \%$ & $3.206 \%$ & 268.47 & 402.20 \\
$5.0 \mathrm{wt} \%$ & $4.439 \%$ & 253.75 & 398.90 \\
\hline
\end{tabular}

elastomers decreased, indicating that the degree of the weight decreasing was greater than the degree of the weight increasing. When the LPCA loading was more than $2.5 \mathrm{wt} \%$, the percentage of the weight change became a negative number. The reason may be that excessive LPCAs hindered the formation of the network of polyurethane. When LPCAWPU elastomers were placed in water, water could enter the network of polyurethane more easily and make more polyurethane dissolved. In a word, LPCAs, or more accurately the mixture of LPCAs and $\mathrm{NaOH}$, increased the water solubility of WPU elastomers.

3.3. Thermal Properties of LPCA-WPU Elastomers. Figure 2 shows thermogravimetric (TG) and derivative thermogravimetric (DTG) curves of LPCA-WPU elastomers with different loadings of LPCAs, while the related data is shown in Table 3. After LPCAs were added into WPU, the weight loss peak of the soft segments of WPU moved to the lower temperature while the weight loss peak of the hard segments almost stayed in the original position, indicating that LPCAs had more influence on the soft segments of WPU rather than on the hard segments. Particularly, when the LPCA loading was $5.0 \mathrm{wt} \%$, the weight loss peak of the soft segments moved further to the lower temperature. Thus, when the LPCA loading was relatively large, the soft segments of WPU were affected more and became easier to decompose.

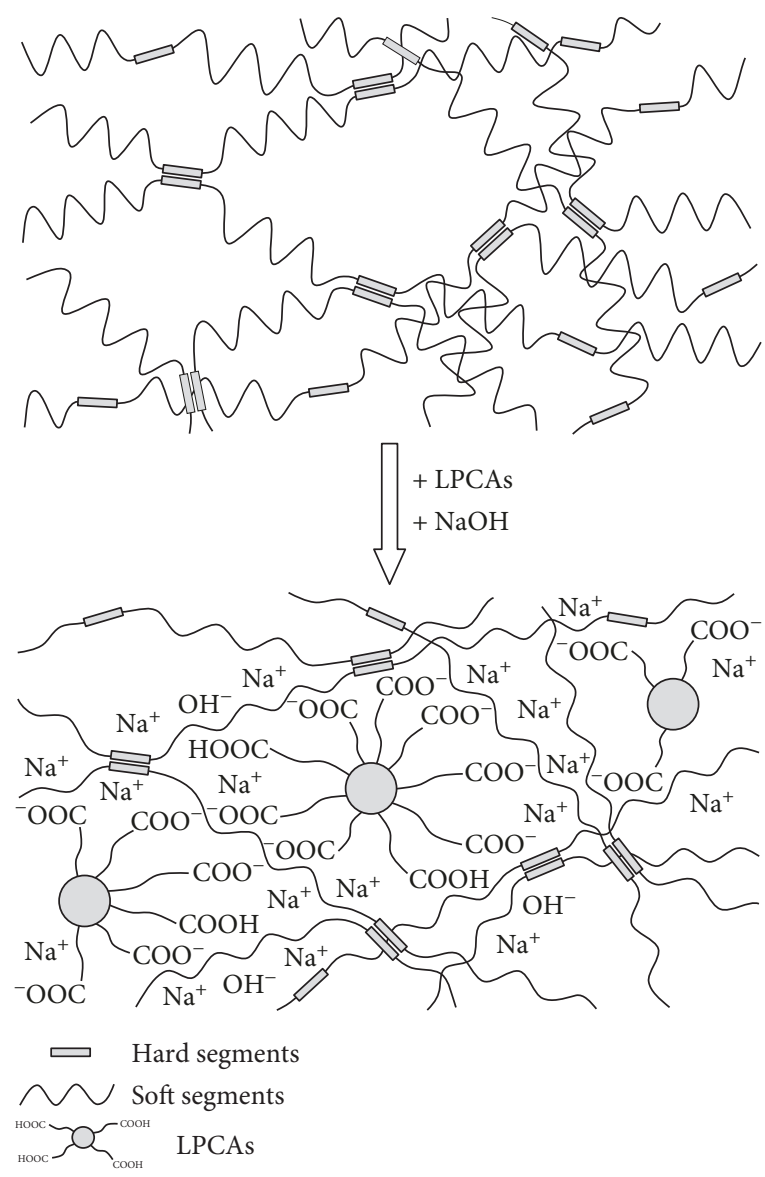

FIGURE 3: Illustration of interactions between LPCAs and WPU elastomers.

3.4. Analysis of the Interactions between LPCAs and WPU Elastomers. Figure 3 illustrates the interactions between LPCAs and WPU elastomers. LPCAs were dissolved in $\mathrm{NaOH}$ solution, and hence, the segments of LPCAs could 


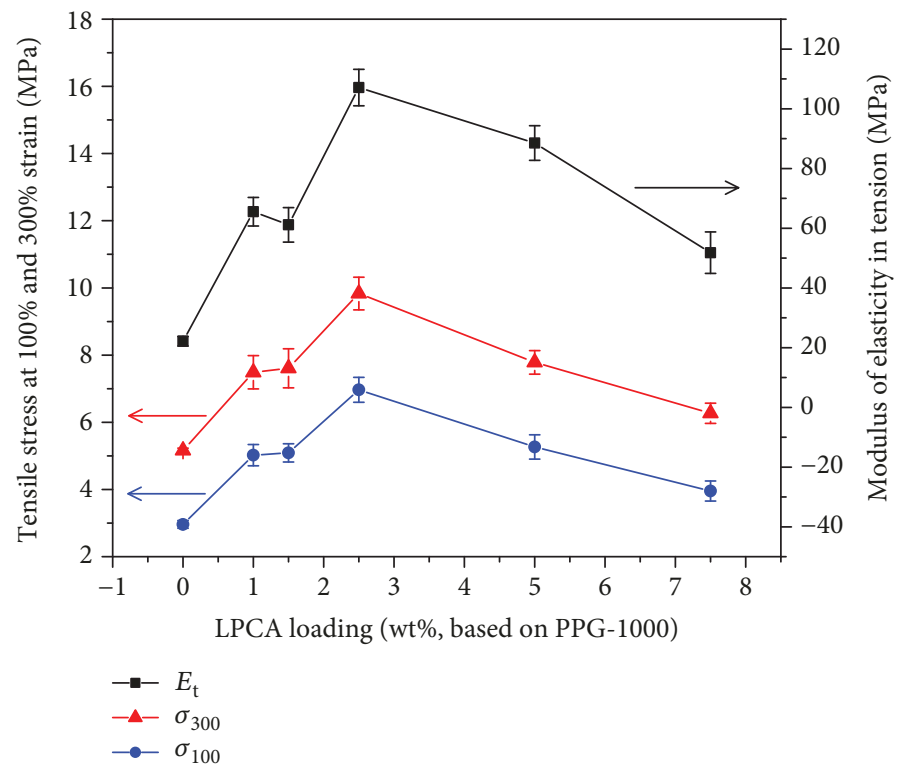

FIGURE 4: Effect of LPCA loading on tensile stress at $100 \%$ strain $\left(\sigma_{100}\right)$, tensile stress at $300 \%$ strain $\left(\sigma_{300}\right)$ and modulus of elasticity in tension $\left(E_{t}\right)$.

fully stretch in water solution. As carboxylic acids are generally weak acids, some ionized carboxyl groups of LPCAs were hydrolyzed to unionized carboxyl groups. After LPCAs were added into WPU in emulsification process, they tended to get into the soft segment regions of WPU and enlarged the interspace of the soft segment regions. LPCAs had a relatively high content of carboxyl, which increased their polarity and helped to interact with the soft segments of WPU via hydrogen bonds. The larger interspace of the soft segment regions led to an increase of the water solubility of WPU. Thus, more water could be retained in the soft segments after the water emulsion of LPCA-WPU was dried. Moreover, as lignin is not thermal stable above $150^{\circ} \mathrm{C}$ [26], when the LPCA-WPU elastomers were heated under a nitrogen atmosphere, LPCAs started to decompose at about $150^{\circ} \mathrm{C}$. The decomposition products of LPCAs enlarged the interspace of the soft segment regions further. The reasons above made the soft segments of the LPCA-WPU elastomers decompose more easily at a lower temperature. Besides, van der Waals forces also contributed the interactions between LPCAs and the soft segments.

3.5. Mechanical Properties of LPCA-WPU Elastomers. Figure 4 and Table 4 show the effect of LPCAs on tensile stress at $100 \%$ strain $\left(\sigma_{100}\right)$, tensile stress at $300 \%$ strain $\left(\sigma_{300}\right)$, and modulus of elasticity in tension $\left(E_{\mathrm{t}}\right)$ of LPCAWPU elastomers. As a kind of modification products of lignin, LPCAs remain some rigid structures of lignin like benzene rings and $\beta-\beta$ linkages [27]. LPCAs hindered the movements of the soft segments of WPU, resulting in an increase of $\sigma_{100}, \sigma_{300}$, and $E_{\mathrm{t}}$ of LPCA-WPU elastomers. When the LPCA loading was $2.5 \mathrm{wt} \%, \sigma_{100}, \sigma_{300}$, and $E_{\mathrm{t}}$ reached top and increased $135.4 \%, 90.5 \%$, and $383.6 \%$ separately comparing with the blank WPU elastomer. However, the hindering effect of LPCAs on the movements of
TABLe 4: Tensile stress at $100 \%$ strain $\left(\sigma_{100}\right)$, tensile stress at $300 \%$ strain $\left(\sigma_{300}\right)$ and modulus of elasticity in tension $\left(E_{\mathrm{t}}\right)$ of LPCAWPU elastomers with different loadings of LPCAs.

\begin{tabular}{lccc}
\hline LPCA loading & $\sigma_{100}(\mathrm{MPa})$ & $\sigma_{300}(\mathrm{MPa})$ & $E_{\mathrm{t}}(\mathrm{MPa})$ \\
\hline $0 \mathrm{wt} \%$ & $2.96 \pm 0.12$ & $5.16 \pm 0.07$ & $22.2 \pm 1.6$ \\
$1.0 \mathrm{wt} \%$ & $5.02 \pm 0.32$ & $7.49 \pm 0.49$ & $65.5 \pm 4.8$ \\
$1.5 \mathrm{wt} \%$ & $5.09 \pm 0.27$ & $7.61 \pm 0.58$ & $61.1 \pm 5.8$ \\
$2.5 \mathrm{wt} \%$ & $6.97 \pm 0.37$ & $9.83 \pm 0.49$ & $107.1 \pm 6.1$ \\
$5.0 \mathrm{wt} \%$ & $5.26 \pm 0.36$ & $7.78 \pm 0.35$ & $88.5 \pm 5.8$ \\
$7.5 \mathrm{wt} \%$ & $3.95 \pm 0.30$ & $6.27 \pm 0.30$ & $51.8 \pm 7.0$ \\
\hline
\end{tabular}

the soft segments could not increase infinitely, and thus, the raising effect of LPCAs on the mechanical properties was limited. As the discussion in Section 3.2, when the LPCA loading was more than $2.5 \mathrm{wt} \%$, the formation of the network of polyurethane was hindered. Less formation of the network caused the decrease of the mechanical properties. Therefore, when the LPCA loading was more than $2.5 \mathrm{wt} \%, \sigma_{100}, \sigma_{300}$, and $E_{\mathrm{t}}$ decreased.

Figure 5 and Table 5 show the effect of LPCAs on tensile strength $\left(\sigma_{\mathrm{M}}\right)$ and tensile strain at break $\left(\varepsilon_{\mathrm{B}}\right)$ of LPCA-WPU elastomers. After LPCAs were added, the interspace of the soft segment regions was enlarged and the soft segments were straightened to a certain degree. The moving space of the soft segments decreased and thus $\varepsilon_{\mathrm{B}}$ decreased at different degrees. For reasons similar to $\sigma_{100}$ and $\sigma_{300}, \sigma_{\mathrm{M}}$ first increased and then decreased in general when the LPCA loading increased.

\section{Conclusion}

Lignin was oxidized to prepare LPCAs, which was later introduced into WPU elastomers and exhibited modification 


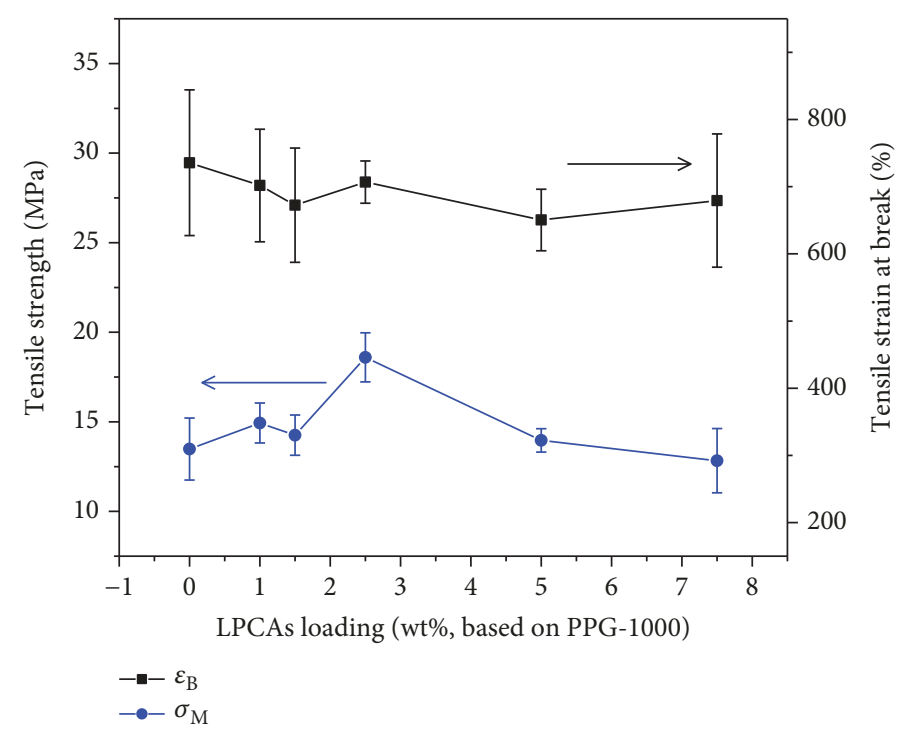

FIgURE 5: Effect of LPCA loading on tensile strength $\left(\sigma_{\mathrm{M}}\right)$ and tensile strain at break $\left(\varepsilon_{\mathrm{B}}\right)$.

TABLE 5: Tensile strength $\left(\sigma_{\mathrm{M}}\right)$ and tensile strain at break $\left(\varepsilon_{\mathrm{B}}\right)$ of LPCA-WPU elastomers with different loadings of LPCAs.

\begin{tabular}{lcc}
\hline LPCA loading & $\sigma_{\mathrm{M}}(\mathrm{MPa})$ & $\varepsilon_{\mathrm{B}}(\%)$ \\
\hline $0 \mathrm{wt} \%$ & $13.48 \pm 1.73$ & $736 \pm 108$ \\
$1.0 \mathrm{wt} \%$ & $14.93 \pm 1.12$ & $702 \pm 84$ \\
$1.5 \mathrm{wt} \%$ & $14.25 \pm 1.12$ & $673 \pm 85$ \\
$2.5 \mathrm{wt} \%$ & $18.60 \pm 1.37$ & $707 \pm 31$ \\
$5.0 \mathrm{wt} \%$ & $13.96 \pm 0.65$ & $650 \pm 46$ \\
$7.5 \mathrm{wt} \%$ & $12.83 \pm 1.79$ & $679 \pm 99$ \\
\hline
\end{tabular}

effects on many aspects of properties. Water resistance test suggested that LPCAs increased the water solubility of WPU elastomers. DTG curves of LPCA-WPU elastomers showed that the weight loss peak of the soft segments of WPU moved to the lower temperature after LPCAs were added. LPCAs made the soft segments decompose more easily, indicating that LPCAs enlarged the interspace of the soft segment regions. The relatively high content of carboxyl helped LPCAs interact with the soft segments. LPCAs hindered the movements of the soft segments, resulting in an increase of the mechanical properties of WPU elastomers. Comparing with the blank WPU elastomer, when the LPCA loading was $2.5 \mathrm{wt} \%$ (based on PPG-1000), $\sigma_{100}$ and $\sigma_{300}$ of the LPCA-WPU elastomers increased $135.4 \%$ and $90.5 \%$ separately, and $E_{\mathrm{t}}$ increased $383.6 \%$. LPCAs can be used as reinforcement fillers of WPU elastomers and can increase the degradability of them, which contributes to develop more functional and eco-friendly WPU materials. Besides, as a kind of bioresource, lignin is utilized further and its usage is broadened.

\section{Data Availability}

All relevant data are within the paper.

\section{Conflicts of Interest}

The authors declare that there are no conflicts of interest.

\section{Acknowledgments}

This work was partly supported by the National Natural Science Foundation of China (no. 51502108), the Foundation of Jilin Province Development and Reform Commission, China (no. 2014N145), the Science and Technology Innovation "Double Ten Project" (no. 55SS06), and the Changchun Science and Technology Bureau (no. 15SS06).

\section{References}

[1] P. Król, "Synthesis methods, chemical structures and phase structures of linear polyurethanes. Properties and applications of linear polyurethanes in polyurethane elastomers, copolymers and ionomers," Progress in Materials Science, vol. 52, no. 6, pp. 915-1015, 2007.

[2] D. P. Pfister, Y. Xia, and R. C. Larock, "Recent advances in vegetable oil-based polyurethanes," ChemSusChem, vol. 4, no. 6, pp. 703-717, 2011.

[3] D. K. Chattopadhyay and K. V. S. N. Raju, "Structural engineering of polyurethane coatings for high performance applications," Progress in Polymer Science, vol. 32, no. 3, pp. 352-418, 2007.

[4] X. Cao, H. Dong, and C. M. Li, "New nanocomposite materials reinforced with flax cellulose nanocrystals in waterborne polyurethane," Biomacromolecules, vol. 8, no. 3, pp. 899-904, 2007.

[5] G. M. Wu, G. F. Liu, J. Chen, and Z. W. Kong, "Preparation and properties of thermoset composite films from twocomponent waterborne polyurethane with low loading level nanofibrillated cellulose," Progress in Organic Coatings, vol. 106, pp. 170-176, 2017.

[6] T. V. Travinskaya, A. N. Brykova, Y. V. Savelyev, N. V. Babkina, and V. I. Shtompel, "(Bio)degradable ionomeric polyurethanes based on xanthan: synthesis, properties, and 
structure," International Journal of Polymer Science, vol. 2017, Article ID 8632072, 10 pages, 2017.

[7] H. Kim, Y. Miura, and C. W. Macosko, "Graphene/ polyurethane nanocomposites for improved gas barrier and electrical conductivity," Chemistry of Materials, vol. 22, no. 11, pp. 3441-3450, 2010.

[8] Y. Tong, S. Bohm, and M. Song, "The capability of graphene on improving the electrical conductivity and anti-corrosion properties of polyurethane coatings," Applied Surface Science, vol. 424, pp. 72-81, 2017.

[9] Q. Kuang, D. Zhang, J. C. Yu et al., "Toward record-high stiffness in polyurethane nanocomposites using aramid nanofibers," Journal of Physical Chemistry C, vol. 119, no. 49, pp. 27467-27477, 2015.

[10] T. N. Tallman, S. Gungor, K. W. Wang, and C. E. Bakis, "Tactile imaging and distributed strain sensing in highly flexible carbon nanofiber/polyurethane nanocomposites," Carbon, vol. 95, pp. 485-493, 2015.

[11] A. Ratnakar, K. Hari Prasad, S. Vivekananthan, P. C. Karthika, and A. Kumar, "Synthesis and characterization of polyurethane-titanium dioxide-hydroxyapatite nanocomposite for biomedical applications," Materials Today: Proceedings, vol. 3, no. 10, pp. 4052-4057, 2016.

[12] J. D. Mangadlao, H. Xu, E. Baer, and R. C. Advincula, "In situ photogeneration of palladium nanoparticles in thermoplastic polyurethane: photopatterning and enhanced oxygen barrier property," Macromolecular Chemistry and Physics, vol. 218, no. 21, 2017.

[13] G. Cui, H. Fan, W. Xia, F. Ai, and J. Huang, "Simultaneous enhancement in strength and elongation of waterborne polyurethane and role of star-like network with lignin core," Journal of Applied Polymer Science, vol. 109, no. 1, pp. 56-63, 2008.

[14] J. H. Lora and W. G. Glasser, "Recent industrial applications of lignin: a sustainable alternative to nonrenewable materials," Journal of Polymers and the Environment, vol. 10, no. 1-2, pp. 39-48, 2002.

[15] H. Lange, S. Decina, and C. Crestini, "Oxidative upgrade of lignin - recent routes reviewed," European Polymer Journal, vol. 49, no. 6, pp. 1151-1173, 2013.

[16] S. H. Ghaffar and M. Fan, "Structural analysis for lignin characteristics in biomass straw," Biomass \& Bioenergy, vol. 57, no. 10 , pp. 264-279, 2013.

[17] S. Laurichesse and L. Avérous, "Chemical modification of lignins: towards biobased polymers," Progress in Polymer Science, vol. 39, no. 7, pp. 1266-1290, 2014.

[18] A. R. Gonçalves and P. Benar, "Hydroxymethylation and oxidation of organosolv lignins and utilization of the products," Bioresource Technology, vol. 79, no. 2, pp. 103-111, 2001.

[19] D. Kai, M. J. Tan, P. L. Chee, Y. K. Chua, Y. L. Yap, and $\mathrm{X}$. J. Loh, "Towards lignin-based functional materials in a sustainable world," Green Chemistry, vol. 18, no. 5, pp. 11751200, 2016.

[20] B. M. Upton and A. M. Kasko, "Strategies for the conversion of lignin to high-value polymeric materials: review and perspective," Chemical Reviews, vol. 116, no. 4, pp. 2275-2306, 2016.

[21] C. Crestini, M. Crucianelli, M. Orlandi, and R. Saladino, "Oxidative strategies in lignin chemistry: a new environmental friendly approach for the functionalisation of lignin and lignocellulosic fibers," Catalysis Today, vol. 156, no. 1-2, pp. 8-22, 2010.
[22] W. He, W. Gao, and P. Fatehi, "Oxidation of kraft lignin with hydrogen peroxide and its application as a dispersant for kaolin suspensions," ACS Sustainable Chemistry \& Engineering, vol. 5, no. 11, pp. 10597-10605, 2017.

[23] S. Laurichesse, C. Huillet, and L. Avérous, "Original polyols based on organosolv lignin and fatty acids: new bio-based building blocks for segmented polyurethane synthesis," Green Chemistry, vol. 16, no. 8, pp. 3958-3970, 2014.

[24] N. Mahmood, Z. Yuan, J. Schmidt, and C. (. C.). Xu, "Depolymerization of lignins and their applications for the preparation of polyols and rigid polyurethane foams: a review," Renewable \& Sustainable Energy Reviews, vol. 60, pp. 317-329, 2016.

[25] S. X. Ren, G. Q. Jiang, and H. J. Qu, Tutorials on Chemical Experiments of Plant Fibers, Northeast Forestry University Press, Harbin, 2008.

[26] V. Fierro, V. Torné-Fernández, D. Montané, and A. Celzard, "Study of the decomposition of kraft lignin impregnated with orthophosphoric acid," Thermochimica Acta, vol. 433, no. 1-2, pp. 142-148, 2005.

[27] W. G. Forsythe, M. D. Garrett, C. Hardacre, M. Nieuwenhuyzen, and G. N. Sheldrake, "An efficient and flexible synthesis of model lignin oligomers," Green Chemistry, vol. 15, no. 11, pp. 3031-3038, 2013. 


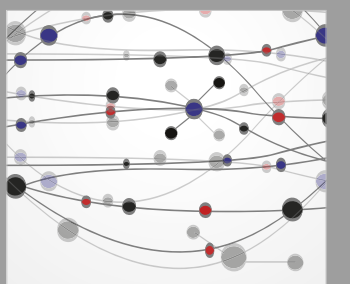

The Scientific World Journal
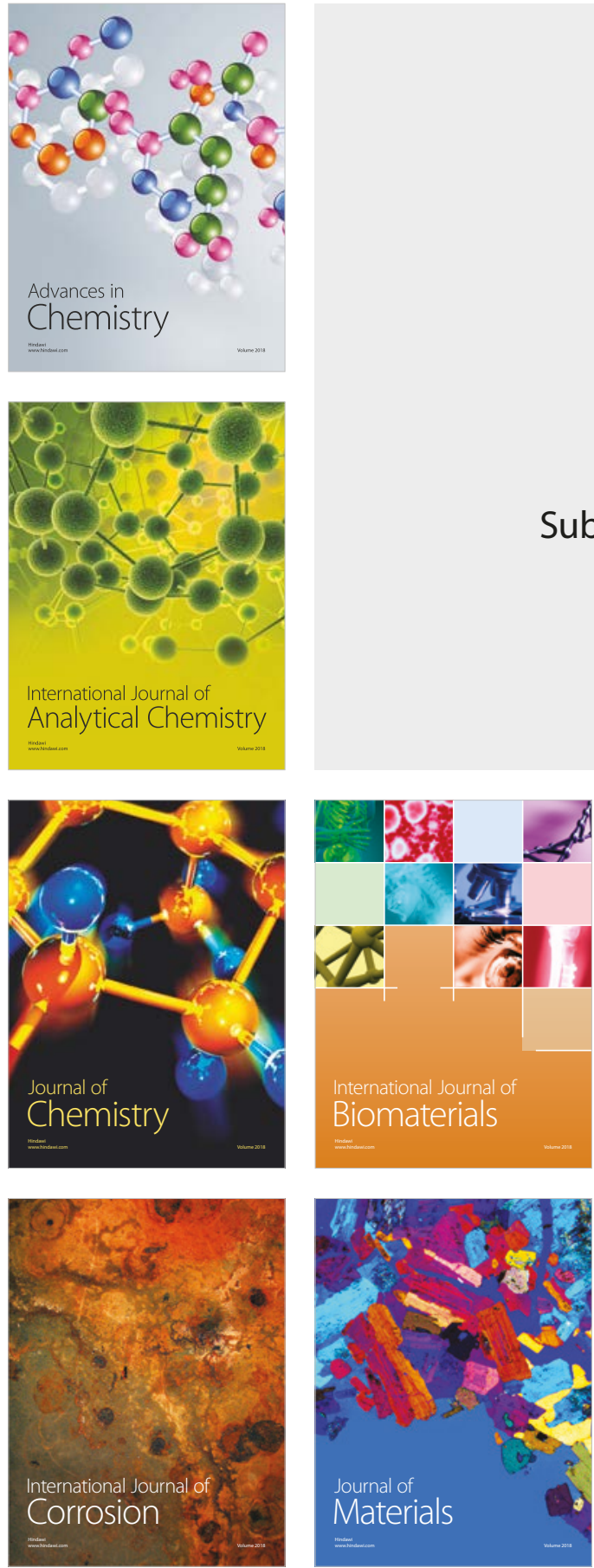

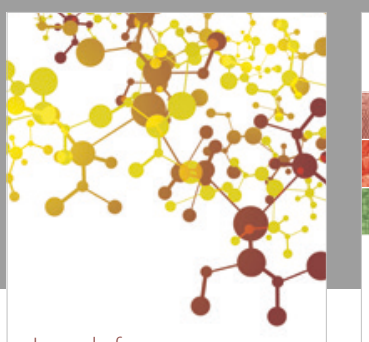

Journal of

Applied Chemistry
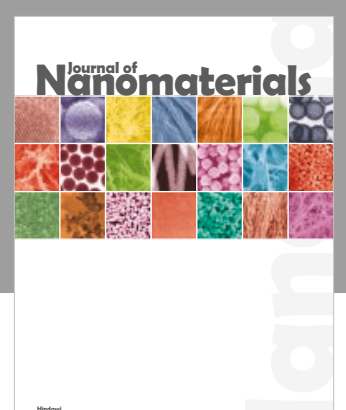

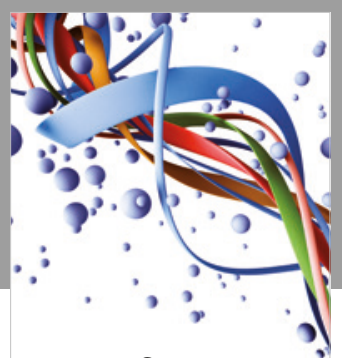

Scientifica

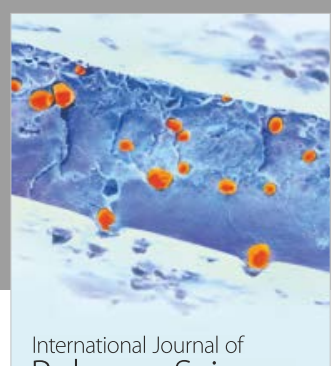

Polymer Science

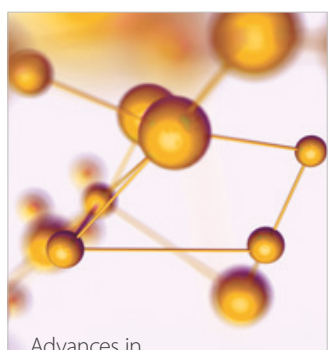

Physical Chemistry
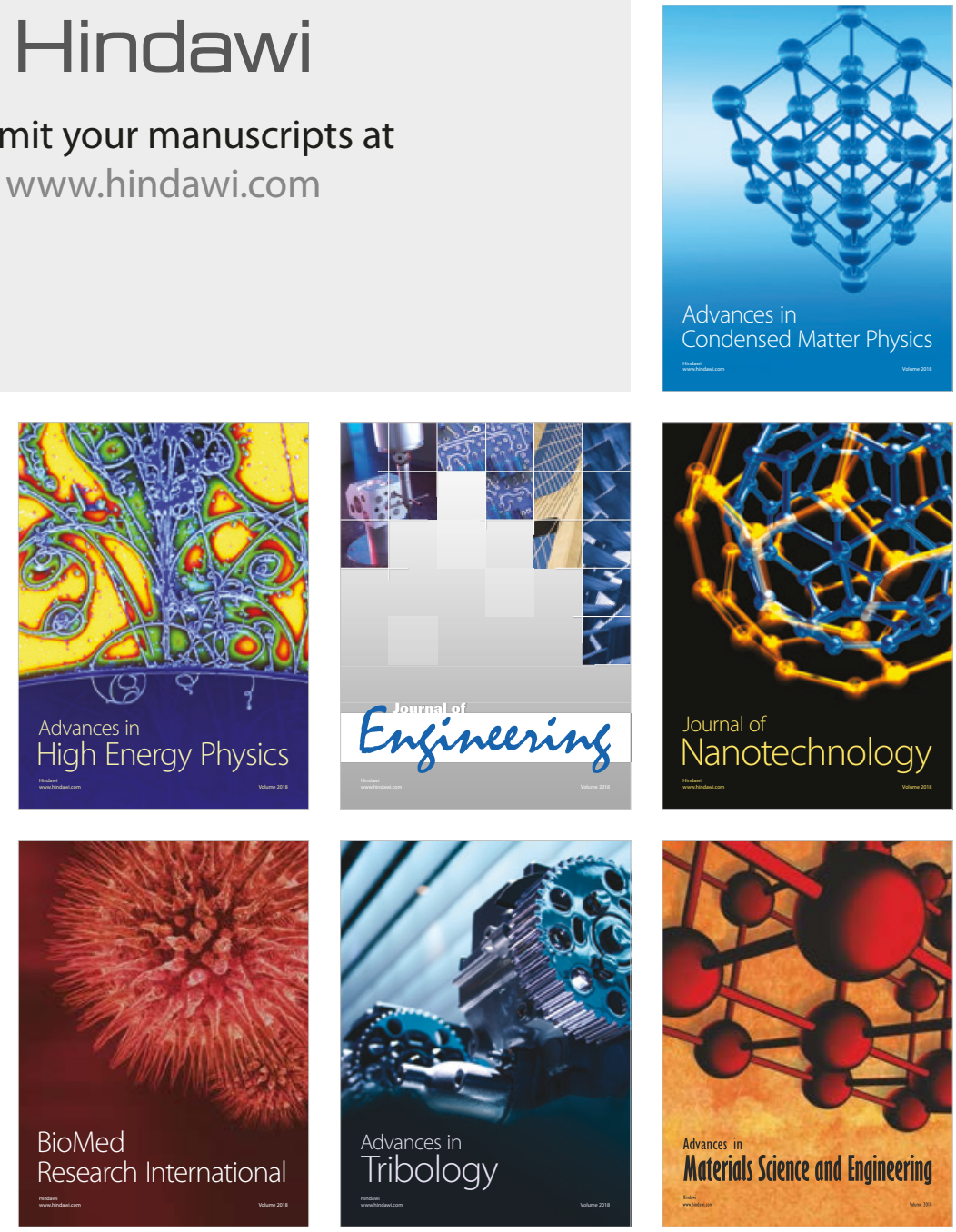